\title{
An overview of the genetic basis of cardiovascular disease
}

\author{
N Laing, ${ }^{1}$ MSc; S M Kraus, ${ }^{2,3}$ FCP (SA); G Shaboodien, ${ }^{3}$ PhD; N A B Ntusi, ${ }^{2,3,4}$ FCP (SA), MD, DPhil \\ ${ }^{1}$ Division of Human Genetics, Department of Medicine, Faculty of Health Sciences, University of Cape Town and Groote Schuur Hospital, \\ Cape Town, South Africa \\ ${ }^{2}$ Division of Cardiology, Department of Medicine, Faculty of Health Sciences, University of Cape Town and Groote Schuur Hospital, \\ Cape Town, South Africa \\ ${ }^{3}$ The Cardiovascular Genetics Laboratory, Hatter Institute of Cardiovascular Research in Africa, Department of Medicine, \\ Faculty of Health Sciences, University of Cape Town, South Africa \\ ${ }^{4}$ Cape Universities Body Imaging Centre, Faculty of Health Sciences, University of Cape Town, South Africa
}

Corresponding author: N A B Ntusi (ntobeko.ntusi@uct.ac.za)

Cardiovascular disease (CVD) is the leading cause of mortality globally. CVD comprises a wide range of clinical entities, i.e. cardiomyopathies, hypertension, aortopathies, coronary artery disease, valvular heart disease, congenital heart disease, dyslipidaemia and arrhythmias, which are heritable. In the past 5 decades, substantial effort has been invested in understanding the genes and specific DNA sequence variants responsible for this heritability. The explosion of new knowledge of genetics and genomics of rare and common forms of CVD has provided a framework for precision cardiology. In this brief review, we summarise the current state of concepts and knowledge of the genetic basis of CVD and reflect on the lessons learnt regarding monogenic, complex polygenic and common forms of CVD. We highlight the pitfalls in interpretation of genetic variants and explore the challenging journey of gene discovery - from genomic localisation to mechanistic insights and finally developing therapies based on identified targets. We discuss the impact of next-generation sequencing and the role of functional studies in understanding the mechanisms by which genetic variation contributes to CVD. Finally, we recommend an approach to genetic testing and counselling for affected individuals and their family members, who may be at risk of developing CVD.

S Afr Med J 2019;109(6):364-370. DOI:10.7196/SAMJ.2019.v109i6.14069

Cardiovascular disease (CVD) claims $\sim 20$ million lives worldwide annually, accounting for a third of global deaths. More than $75 \%$ of deaths from CVD occur in low- and middle-income countries (LMICs). Eighty-five percent of all CVD deaths worldwide are due to myocardial infarction (MI) and stroke. ${ }^{[1]}$ In sub-Saharan Africa (SSA), heart failure is the dominant form of CVD; the causes are largely non-ischaemic, i.e. hypertension, cardiomyopathy, rheumatic heart disease, pericardial disease and cor pulmonale. ${ }^{[2]}$ The high prevalence and changing profile of CVD in SSA are directly linked to population dynamics and epidemiological transition in some of the most vulnerable societies. The epidemiological transition, characterised by an increase in obesity, hypertension and diabetes mellitus, has seen an increase in non-communicable diseases (NCDs) in urban, semi-urban and rural areas. The burden of NCDs is likely to increase further, as antiretroviral therapy reduces mortality and affects the cardiovascular system. Further, infections and NCDs are fuelled by common precursors such as poverty and poor education. ${ }^{[3]}$ CVD related to genetic predisposition is gaining increased awareness in SSA owing to advances in genetic technologies, resulting in substantial expansion of knowledge of the genetic basis and mechanisms of CVD. ${ }^{[4]}$

Table 1 provides a glossary of the genetic terminology used in this article..$^{[5]}$

\section{What are genetic variations?}

Each human being is unique, but genetically, we are $99.9 \%$ the same. The remaining $0.1 \%$ is responsible for all the differences that make each one of us individual and is caused by genetic variation (GV). $\mathrm{GV}$ is the difference in DNA sequences between individuals within a population and can be caused by repair errors in the replication machinery. Variation occurs in germ cells and somatic cells. Only variation that arises in germ cells can be inherited from one individual by another and affect population dynamics, and ultimately evolution. Large changes to our genome may have no effect at all, whereas the change of a single nucleotide can have a huge impact. Mutations and recombination are major sources of variation.

There are three types of GV: (i) single-nucleotide polymorphisms (SNPs) are the most common, occurring about once every 1000 bases; (ii) insertion or deletion of a single stretch of DNA sequence that can range from two to hundreds of base-pairs in length; and (iii) genetic rearrangements that can affect several genes or large areas of a chromosome at once. GV includes both copy number variation and chromosomal rearrangement events.

Most variation is meaningless and does not affect our ability to survive or adapt. For example: (i) 'silent mutations' in DNA, which change the DNA, but not the amino acid sequence; (ii) other mutations may change the amino acid sequence of a protein, but not the overall function of that protein; and (iii) other polymorphisms do not seem to affect our survival at all. Other variations are positive and improve our ability to survive or adapt, e.g. mutations that cause sickle cell anaemia have a protective effect against malaria. Some variation leads to disease such as monogenic single-gene disorders, e.g. the inherited cardiomyopathies.

The effect of GVs on phenotype (effect size) follows a gradient from none (indiscernible) to large (clinically consequential). The vast majority of GVs do not have significant biological effects. GVs that cause large phenotypic effects are usually responsible for single-gene disorders that exhibit Mendelian patterns of inheritance. Complex and polygenic disorders are primarily due to many common GVs, each exerting small, indiscernible effects, but collectively and through interactions with non-genetic factors, influencing the risk of the complex phenotype, e.g. coronary artery disease (CAD). GVs are 


\section{Table 1. Glossary of genetic terms used}

\begin{tabular}{|c|c|}
\hline Allele & $\begin{array}{l}\text { An allele is one of the possible forms of a gene. Most genes have two alleles, a dominant allele } \\
\text { and a recessive allele. If an organism is heterozygous for that trait, or possesses one of each } \\
\text { allele, then the dominant trait is expressed. A recessive allele is only expressed if an organism is } \\
\text { homozygous for that trait, or possesses two recessive alleles. Alleles were first defined by } \\
\text { Gregor Mendel in the law of segregation }\end{array}$ \\
\hline Exome & $\begin{array}{l}\text { The DNA that is coded for in the exons of genes that code for proteins, i.e the 'protein-coding' } \\
\text { regions of the genome }\end{array}$ \\
\hline Expressivity & The degree to which a trait is expressed in an individual, i.e. the intensity of the phenotype \\
\hline Founder & $\begin{array}{l}\text { A common mutation that occurs in higher frequency in a specific population due to a common } \\
\text { ancestor }\end{array}$ \\
\hline Genome & All the DNA contained in an organism or a cell, including nuclear and mitochondrial DNA \\
\hline Genotype & The set of genes in our DNA that is responsible for a particular trait \\
\hline Genome-wide association study & $\begin{array}{l}\text { Also known as whole-genome association study, is an observational study of a genome-wide set of } \\
\text { genetic variants in different individuals to see if any variant is associated with a trait }\end{array}$ \\
\hline Haplotype & $\begin{array}{l}\text { A haplotype (haploid genotype) is a group of alleles in an organism that are inherited together from } \\
\text { a single parent }\end{array}$ \\
\hline Heterogeneity & $\begin{array}{l}\text { Genetic heterogeneity occurs when mutations at two or more genetic loci produce the same or } \\
\text { similar phenotypes (either biochemical or clinical) }\end{array}$ \\
\hline Homozygosity & $\begin{array}{l}\text { Genetic homozygosity describes a situation where an individual inherits the same alleles for } \\
\text { a particular gene from both parents }\end{array}$ \\
\hline Locus & $\begin{array}{l}\text { A locus (plural loci) in genetics is a fixed position on a chromosome, such as the position of } \\
\text { a gene or a marker (genetic marker) }\end{array}$ \\
\hline Mendelian genetics & $\begin{array}{l}\text { A Mendelian trait is one that is controlled by a single locus in an inheritance pattern. In such cases, } \\
\text { a mutation in a single gene can cause a disease that is inherited according to Mendel's laws. Examples } \\
\text { include sickle cell anaemia and cystic fibrosis. Mendel's studies yielded three 'laws' of inheritance: } \\
\text { the law of dominance, the law of segregation and the law of independent assortment. Each of these } \\
\text { can be understood through examining the process of meiosis }\end{array}$ \\
\hline Mendelian randomisation & $\begin{array}{l}\text { A method of using measured variation in genes of known function to examine the causal effect of } \\
\text { a modifiable exposure on disease in observational studies }\end{array}$ \\
\hline Penetrance & The probability of a gene or trait being expressed \\
\hline Phenotype & The physical expression, or characteristics, of that trait due to a specific genotype \\
\hline Pleiotropy & The ability of a single gene to influence multiple phenotypic traits \\
\hline
\end{tabular}

classified functionally as pathogenic, likely pathogenic, of uncertain significance, likely benign and benign (Table 2).

\section{Genetic cardiovascular conditions}

CVD is caused by both genetic and environmental factors. In every condition, the interaction of genes with the environment is complex and profoundly affects phenotype. Genetic cardiovascular conditions may show incomplete or age-related penetrance, whereby individuals may not show signs of the condition until a certain age, or until environmental or lifestyle factors precipitate the manifestation of physical signs of the disease, e.g. hypertrophic cardiomyopathy (HCM) usually manifests in early adolescence during periods of rapid somatic growth. Genetic CVDs also demonstrate variable expressivity, whereby different family members with the same genetic mutation express the disease in different ways (i.e. varying severity). Expressivity is dependent upon environmental, lifestyle and background genetic modulators. Genetic cardiovascular conditions demonstrate clinical and genetic overlap, which may complicate the interpretation of genetic test results. Despite these caveats, knowledge of the genetic nature of a particular condition may be important - not only may it inform the need for familial screening and therefore early diagnosis and treatment to delay onset of disease, but it may also aid in risk stratification for sudden cardiac death (SCD) in affected individuals and potentially effect treatment and management decisions. ${ }^{[8]}$

Genetic cardiovascular conditions may be Mendelian (i.e. due to a single-gene defect) or non-Mendelian (i.e. due to complex, common
GVs), or mitochondrial (Table 3). Most of these conditions display significant heterogeneity, i.e. locus, allelic and phenotypic. Locus heterogeneity refers to the phenomenon that numerous genes can result in the same clinical phenotype (e.g. $>20$ genes, mostly of the sarcomere, have been implicated in the aetiology of HCM). Allelic heterogeneity denotes various mutations, or alleles, within a gene, which can cause the same condition. Phenotypic heterogeneity refers to mutations in the same gene causing different phenotypes. For example, MYH7 gene mutations can cause HCM, dilated cardiomyopathy (DCM), left ventricular non-compaction (LVNC) or restrictive cardiomyopathy (RCM) and can also be associated with myopathy. Similarly, SCN5A gene mutations not only cause arrhythmia syndromes, such as long QT syndrome (LQTS), short QT syndrome (SQTS) and Brugada syndrome, but also DCM. This genetic diversity means that many mutations may be 'private', defined by their presence in only single families, with as many as $60-90 \%$ being described as private in the setting of cardiomyopathies. ${ }^{[9]}$ Recurrent or 'founder' mutations for certain conditions have been described, and may have important implications for certain conditions in South Africa (SA) (e.g. LQTS, HCM and familial hypercholesterolaemia $(\mathrm{FH})$ ).

Single-gene disorders may be inherited as autosomal dominant (e.g. HCM, DCM, LVNC), autosomal recessive (e.g. LQTS, arrhythmogenic right ventricular cardiomyopathy (ARVC) with Naxos syndrome) or X-linked (e.g. Duchenne muscular dystrophy with cardiac involvement). Importantly, lack of family history does not imply that 
Table 2. Classification of genetic variants

\begin{tabular}{|c|c|c|c|}
\hline Category & Definition & Utility for proband & Utility for family \\
\hline Pathogenic & $\begin{array}{l}\text { Loss-of-function and pathogenic variants shown to cause } \\
\text { the disease through co-segregation and linkage analysis or } \\
\text { functional studies in large families }\end{array}$ & $\begin{array}{l}\text { Establishes diagnosis, } \\
\text { may inform } \\
\text { management }\end{array}$ & $\begin{array}{l}\text { Can be used for predictive } \\
\text { genetic testing }\end{array}$ \\
\hline Likely pathogenic & $\begin{array}{l}\text { Evidence for the causal role based on statistical enrichment } \\
\text { in small families and trios with the disease. Robust linkage is } \\
\text { hindered by small size of families or sporadic nature of the } \\
\text { disease. To reduce the possibility of random co-segregation } \\
\text { in small families, findings require testing for replication in } \\
\text { independent populations }\end{array}$ & $\begin{array}{l}\text { Possibly suggests } \\
\text { diagnosis, may inform } \\
\text { management or lead to } \\
\text { additional diagnostic } \\
\text { tests }\end{array}$ & $\begin{array}{l}\text { Predictive genetic testing of } \\
\text { unaffected relatives should } \\
\text { be approached with great } \\
\text { caution, may be combined } \\
\text { with phenotypic evaluation } \\
\text { and surveillance }\end{array}$ \\
\hline $\begin{array}{l}\text { Variant of unknown } \\
\text { significance }\end{array}$ & $\begin{array}{l}\text { Case-control studies will show an association between } \\
\text { genetic variants and the phenotype. Replication in an } \\
\text { independent study population is necessary. Disease- } \\
\text { associated variants may be in linkage disequilibrium with } \\
\text { the actual pathogenic variants, requiring additional studies } \\
\text { to confirm actual pathogenicity }\end{array}$ & Unknown & $\begin{array}{l}\text { Should not be used for } \\
\text { predictive genetic testing } \\
\text { Testing of affected relatives } \\
\text { for segregation may provide } \\
\text { evidence of causality }\end{array}$ \\
\hline Likely benign & $\begin{array}{l}\text { These variants may affect expression levels, structure and } \\
\text { function of proteins, but have not been associated with } \\
\text { disease }\end{array}$ & None & $\begin{array}{l}\text { No option for predictive } \\
\text { genetic testing, rely on } \\
\text { longitudinal phenotypic } \\
\text { evaluation }\end{array}$ \\
\hline Benign & $\begin{array}{l}\text { Comprises the vast majority of genetic variants in the } \\
\text { genome, which have absolutely no impact on protein } \\
\text { expression, structure and function, and no impact on } \\
\text { phenotype }\end{array}$ & None & $\begin{array}{l}\text { No option for predictive } \\
\text { genetic testing, rely on } \\
\text { longitudinal phenotypic } \\
\text { evaluation }\end{array}$ \\
\hline
\end{tabular}

the condition does not have a genetic basis, as it could represent a de novo mutation, incomplete penetrance or an uninformative family history. ${ }^{[10]}$ Complex traits associated with polygenic states, such as serum low-density lipoprotein (LDL) cholesterol levels and CAD, are extremely common. Therefore, genetic heterogeneity from GVs across the frequency spectrum may be the rule. Consider plasma triglycerides: $\sim 50 \%$ of the inter-individual variability in plasma triglycerides is estimated to be based on GVs. ${ }^{[1]}$

\section{When to suspect an inherited cardiovascullar disease}

There are several key features of CVD that should raise suspicion of a strong genetic component to disease. These include extreme phenotypes: unexplained severe disease or early age of onset (childhood or early adulthood), presence of a family history, no identifiable environmental or other cause for the condition in an otherwise healthy individual (idiopathic), history of premature SCD, and presence of additional organ involvement or other syndromic features. ${ }^{[4]}$

\section{Discovering genes for cardiovascular disease}

Traditionally, two major approaches for gene discovery in CVD have been used, i.e. linkage analysis and genetic association. The choice of approach has depended on the pattern of segregation of disease whether consistent with Mendelian ratios or polygenic complexity. ${ }^{[12]}$ For Mendelian forms of CVD, direct DNA sequencing and/or linkage analysis has successfully yielded causal genes and mutations. In 1985, Lehrman et al. ${ }^{[13]}$ sequenced the LDL receptor gene in a patient with homozygous $\mathrm{FH}$ and uncovered a $5 \mathrm{kB}$ deletion that eliminated several exons in the first demonstration of a mutation for Mendelian CVD. In 1989, linkage analysis was used to localise the chromosomal position of a causal gene for HCM. ${ }^{[14]}$ Since then, there has been a plethora of discoveries for genes responsible for single-gene CVDs.

\section{Lessons learnt from Mendelian forms of cardiovascular disease}

Rare single-gene variants. Since Mendelian diseases are rare, there was initial scepticism regarding whether the genes and mechanisms that cause CVD would inform our understanding of common forms of the disease. Linkage studies have radically transformed our understanding of CVD. Selected examples of Mendelian diseases, the responsible genes, and the gleaned biological and clinical insights are detailed in Table 3. Of note is monogenic severe FH, where the 6 responsible genes have led to fundamental new biological concepts and supported the development of new therapies.

Genotype-phenotype correlations. Since the early discovery of genes responsible for CVD, researchers have been looking for genotype-phenotype correlations to explain the impact of genetic background on physical signs. While there are clear examples where single-gene mutations lead to straightforward genotype-phenotype associations, often other more complex relationships also exist. The complexity arises from 3 distinct genetic phenomena: pleiotropy, penetrance and expressivity (Table 1). Pleiotropy, penetrance, expressivity and non-genetic factors conspire to ensure that even in single-gene disorders, genotype does not 'equal' a specific phenotype, with several important consequences: (i) gene discovery is more difficult, as genotype may not segregate perfectly with phenotype, reducing the power of linkage; (ii) there is intense interest in identifying modifiers - genetic or environmental - that modulate the relationship between genotype and phenotype; and (iii) in many Mendelian diseases, it has been difficult to develop genotype-specific prognostic or therapeutic recommendations. ${ }^{[12]}$

Tenuous path from gene discovery to understanding disease mechanisms and developing targeted treatment. The ability to identify causative mutations is only the beginning of the pathophysiological link. The underlying signalling pathways activated by the gene and protein need to be understood. Ultimately, putative therapeutic 


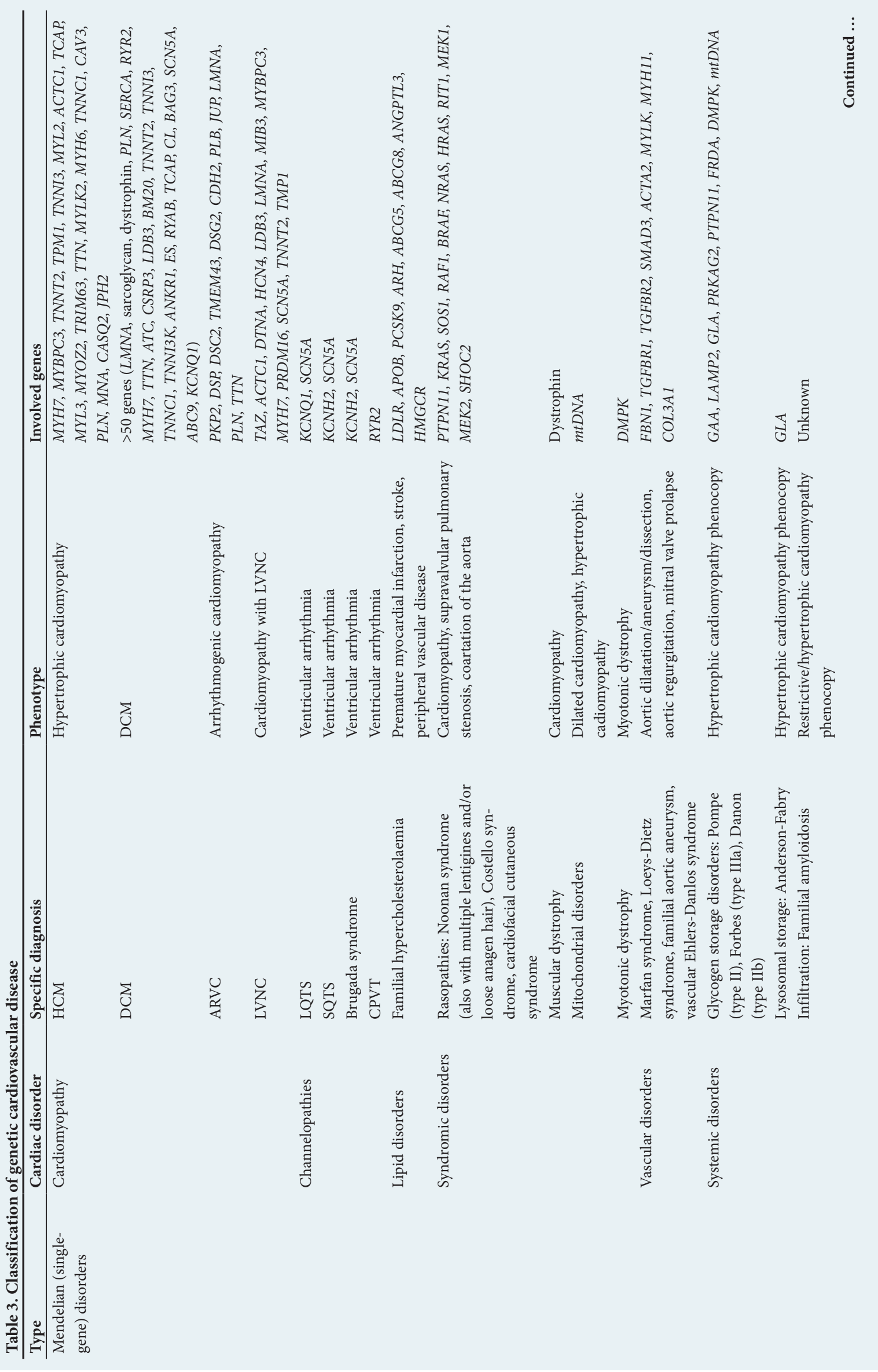




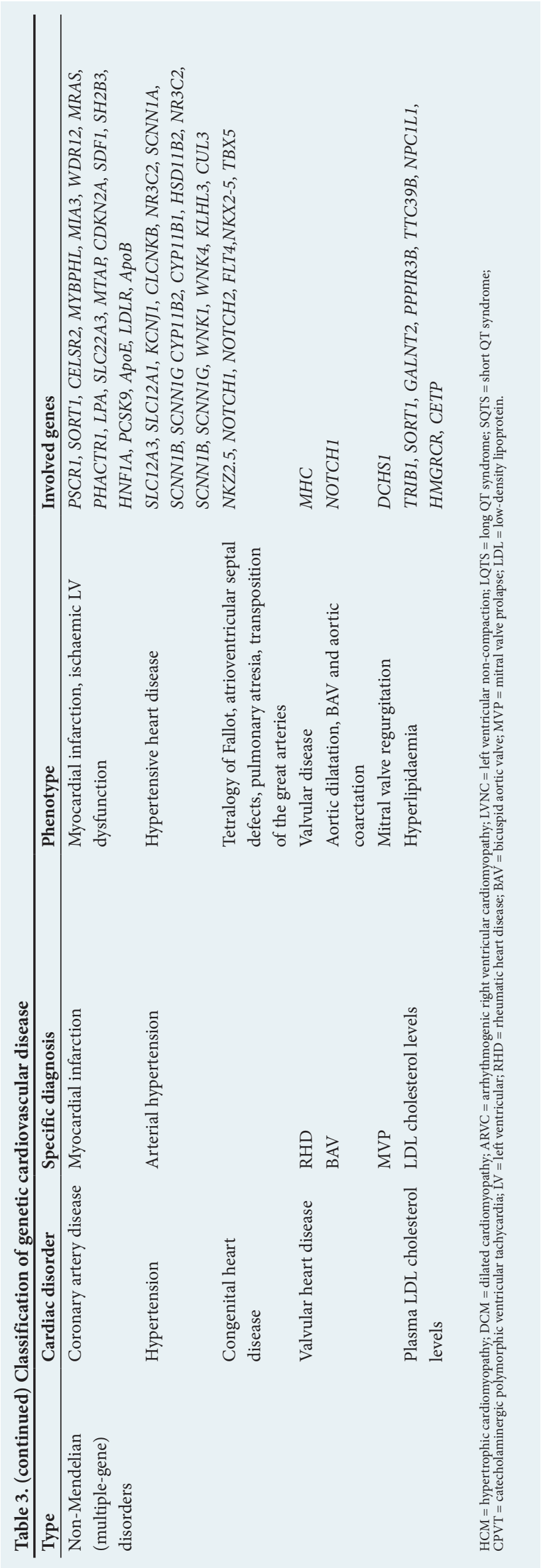

targets must also be exploited. For example, in Marfan's syndrome, the path from the discovery of FBN1 as the causal gene to a breakthrough in the molecular understanding of the disease took more than 2 decades. The importance of the role of transforming growth factor $\beta$ (TGF- $\beta$ ) was not understood until recently. Microfibrils normally bind the large latent TGF- $\beta$ complex, and failure of this to occur results in increased TGF- $\beta$ activation and signalling. Investigators are currently exploring the hypothesis that blocking of TGF- $\beta$ signalling will ameliorate the growth of aortic aneurysms in Marfan's syndrome. ${ }^{[15]}$

\section{Lessons learnt from complex polygenic and common forms of cardiovascular disease}

GVs across the spectrum of allele frequency contribute to most complex and common diseases, as well as quantitative traits. There is a strong overlap between genome-wide association studies (GWAS) and genes identified through Mendelian families, e.g. 19 genes have been identified as monogenic causes of extremely low or high levels of LDL cholesterol, high-density lipoprotein (HDL) cholesterol and triglycerides; loci harbouring 16 of these genes were also mapped using GWAS. Monogenic and polygenic variants often coexist within the same condition.

GWAS. The proportion of overall phenotypic variance explained by a GV may have little correlation with the ultimate therapeutic or biological value of the gene mapped by the GV. Phenotypic variance explained by a variant is a function of two key parameters, i.e. allele frequency and effect size. For Mendelian diseases, the causal variants typically confer large effects but explain a small proportion of trait variance due to their rare frequencies. GVs from GWAS are common but explain only a small proportion of trait variance due to modest effects. Nevertheless, variants that explain a small proportion of phenotypic variance may provide substantial biological or therapeutic insights. For example, GVs in introns of HMGCR confer a small effect on plasma LDL cholesterol; however, targeting of these genes with statins or ezetimibe has a much more dramatic effect on LDL cholesterol. To date, there have been no rare, large-effect Mendelian mutations described in $H M G C R$, presumably because such mutations are highly deleterious and not tolerated. Despite the success of GWAS, most mapped loci have been a challenge to move from genomic localisation to understanding the biological mechanism. The reasons for this observation are manifold: (i) in the context of discovery of Mendelian genes, inferring new biology from human genetics takes time and only about 13 years have elapsed since the initial GWAS publications; (ii) gene mapping and experimental follow-up require unique skill sets, expertise and collaboration; and (iii) genetic mapping by association gives us gene regions and not necessarily specific causal GVs or causal genes.

GVs may distinguish causal from non-causal biomarkers. In 1961, Kannel et al. ${ }^{[16]}$ established an association of plasma total cholesterol with future risk of CAD. Since then, hundreds of soluble biomarkers have similarly been associated with risk of CAD. The important question is how many of these biomarkers directly cause CAD and how many simply reflect other causal processes. Both causal and noncausal biomarkers may predict risk for future disease, but only a causal biomarker may be appropriate as a therapeutic target. In Mendelian randomisation, DNA variants are used to address the question of whether an epidemiological association between a risk factor and disease reflects a causal influence of the former on the latter. ${ }^{[17]}$ If, in an adequately powered sample, the predicted association between the GV and disease were not observed, it would argue against a causal role for the GV in the pathogenesis of the disease. 


\section{Next-generation sequencing approaches}

Next-generation sequencing platforms have markedly decreased the cost of DNA sequencing compared with Sanger sequencing. While whole-exome and whole-genome sequencing have been successfully used to identify new genes for several Mendelian forms of CVD, sequencing just the exome (rather than the entire genome) is justified in the search for genetic causes of rare inherited disorders because most alleles responsible for Mendelian disorders disrupt the protein-coding sequence. The yield of exome sequencing in solving Mendelian disorders is difficult to predict, as negative results have not been routinely reported. ${ }^{[18]}$ It is often difficult to arrive at a single causal mutation after exome sequencing because: (i) the causal GV may not be protein coding (i.e. located in an intron); (ii) the causal GV may be protein coding but the relevant gene not successfully sequenced; (iii) if the causal mutation is not fully penetrant, it will be present in both phenotypically affected and unaffected individuals; (iv) segregation of a phenotype in a family may be due to non-genetic factors (e.g. in a multi-generation family, where multiple individuals are affected with MI, the pattern may be due to a new Mendelian gene or poor lifestyle habits shared by the family); and $(v)$ in contrast to single nucleotide substitutions, methods for identifying small insertion-deletions and copy number changes from short-read sequence data are imprecise. For complex phenotypes (e.g. MI) that arise after decades of pathology, distinguishing functional v. neutral missense mutations is key. While advancements in sequencing techniques, and the development of various pathogenicity prediction tools and publicly available resources on variant frequency in large populations have been informative, clinical applicability remains a challenge.

\section{Functional studies}

Although technological advantages provide cumulative information of genetic causality, the prediction of pathogenicity and the interpretation of clinical consequences of a GV remain challenging. Functional assessment is the most powerful and well-validated strategy to elucidate the role of a candidate variant to the disease pathophysiology, as nothing is more convincing than in vivo data derived from animal models used to characterise the clinical phenotype and the mechanism by which the candidate GVs are shown to cause disease. ${ }^{[19]}$ Knock-in and knock-out animal models have been employed for this purpose for decades, but animal models fail to fully recapitulate the human phenotype.

Use of stem cells and programmed cells for understanding CVD. More recently, human adult somatic cells can be reprogrammed into a pluripotent state by activating differentiation transcription factors. Induced pluripotent stem (iPS) cells can differentiate into the specific cell types affected by disease. iPS cells have provided an unprecedented opportunity to investigate the consequences of human GV on cellular phenotypes that may contribute to disease. iPS cells have contributed enormously to understanding the biology of LQTS, HCM, DCM and Noonan syndrome. The ability to refine functional effects of GVs has been advanced through the use of zinc-finger nucleases and transcription activator-like effector nuclease (TALEN) and clustered regularly interspaced short palindromic repeats (CRISPR)-associated protein 9 (Cas9) technology that specifically introduces or removes GVs in stem cells to reveal the contribution of individual GVs to cellular phenotypes.

Use of zebrafish to study human CVD. Zebrafish possess a variety of features that are advantageous for use as an experimental model organism for the study of CVD. Due to their small size $(2-4 \mathrm{~cm})$, zebrafish are easy to handle and one female can produce 200 eggs per week. Zebrafish embryos develop externally and very rapidly to freely swimming and fed larvae within 5 days. The zebrafish is an excellent system for microscopic applications, as embryos are transparent and numerous transgenic fluorescent reporter lines are available. Because of their suitability for imaging applications, zebrafish are also highly interesting for high-throughput small compound screens. They are ideal for the study of heart development and disease: (i) zebrafish heart development proceeds fast and results in a differentiated twochambered heart within 48 hours postfertilisation; (ii) zebrafish embryos, in contrast to mammalian or avian embryos, are able to cover their oxygen demand by diffusion during the first days of development and are not dependent on blood circulation. This enables the investigation of gene knock-outs or knock-downs, even if they lead to severe defects of the cardiovascular system; and (iii) humans and zebrafish share a $70 \%$ sequence similarity, and $84 \%$ of human disease-causing genes can also be found in the zebrafish genome. The different morphology of the zebrafish heart limits the translation of findings into the mammalian system; without a coronary artery system during embryogenesis, zebrafish show a vasculature on the heart surface, which restricts the study of CAD. Functional verification of candidate genes using CRISPR-Cas9 has supported the role of several genes in the pathophysiology of CVD. Zebrafish hearts maintain their ability to regenerate throughout their lifetime, providing novel insights to understanding human cardiac regeneration. ${ }^{[20]}$

\section{The future of genetic applications in} cardiovascular disease

The widespread use and availability of genetic approaches for the diagnosis of CVD have allowed the study of direct and indirect genetic predisposition, prevention of CVD, genetic testing, population screening and precision cardiology, including pharmacogenetics and pharmacogenomics. In the future, the ability to use specific therapies based on specific genotypes will be widespread (as is already the case in oncology), as will gene therapy for specific singlegene disorders. Barriers to implementing these advances in general cardiology include the inability of physicians to interpret genetic test results and provide appropriate counselling, poor understanding of risk probabilities and growing concern with ethical issues related to emerging genetics and genomics. A major challenge, already evident, is how LMICs will have access to novel and costly research and commercial genetic advances, including genetic screening on a broad scale. A major inequity that needs addressing in the future is the dearth of data on the genetic underpinnings of CVD in SSA. Our group has recently shown that a gene panel with a yield of $>60 \%$ in a European population has a yield of $18 \%$ in South Africans with HCM, highlighting the need to identify genetic causes of CVD in Africans. ${ }^{[2]}$ Similarly, GVs identified in non-SSA populations need to be treated with caution until population screening in local populations has been performed and verified as pathogenic, as it has previously been demonstrated that rare GVs in European populations could represent common GVs in African populations.

\section{Genetic testing recommendations and genetic counselling}

Different types of genetic testing include pre-implantation testing, diagnostic testing at any age, carrier testing, pre-symptomatic testing, postmortem testing, pharmacogenomic testing, risk-prediction testing and direct-to-consumer testing. Importantly, all genetic testing should be based on careful case definition and accurate 
phenotyping. In patients suspected of having familial disease, the first step is to construct a family pedigree, which often informs the pattern of inheritance. If the pedigree suggests a Mendelian pattern of inheritance (e.g. autosomal dominant, autosomal recessive or $\mathrm{X}$-linked recessive), cascade clinical screening is recommended. After informed consent and a comprehensive counselling process by a qualified professional, DNA should be obtained from the proband (clinically affected index patient) for genetic testing. If a causative GV is identified, it can be useful in: (i) clarifying the diagnosis in a person who has or is suspected of having CVD; (ii) identifying the cause of CVD in a family; (iii) providing options for family planning, including pre-implantation genetic diagnosis to avoid having affected offspring; and (iv) predicting which family members are at increased risk of developing CVD by offering predictive testing to unaffected family members. Those family members who have the mutation will require close medical follow-up, as they are at increased risk of developing CVD. Individuals who test negative for the mutation are unlikely to develop the disease and, therefore, do not usually require clinical follow-up and can be reassured. Successful predictive testing in families relies on a high degree of certainty that the GV identified is pathogenic and considered causal of the CVD in a specific family. Interpretation of genetic testing warrants caution. For example, a negative result in a proband means that the laboratory has been unable to identify a disease-causing mutation in the genes evaluated. It is nevertheless possible that the cause of the CVD is genetic. Similarly, the discovery of new GVs should be viewed with caution until these have been subjected to functional testing in large family pedigrees or in independent populations. The physician must always be prepared for the wide range of emotional reactions, including anger, guilt, grief, sadness, fear and anxiety, which accompany a new genetic diagnosis or misdiagnosis.

\section{Conclusions}

Technological advances are unravelling the genetic underpinnings of CVD and have ushered in a new era of genetically informed diagnosis of both rare and common forms of CVD. It is important for clinicians to understand the capabilities and limitations of genetic and genomic testing in different clinical settings and to translate these into clinical practice.

\section{Declaration. None.}

Acknowledgements. We gratefully acknowledge our patients and their families who teach us daily about the complexities of the genetics of cardiovascular disease.
Author contributions. NL and NABN drafted the manuscript; GS and SMK critically reviewed it and gave input into the writing; and NABN approved the changes and takes responsibility for the content.

Funding. Prof. NAB Ntusi gratefully acknowledges funding support from the National Research Foundation, South African Medical Research Council, GlaxoSmithKline, Newton Foundation (UK), Technology Innovation Fund, Harry Crossley Foundation, American Heart Association, US National Institutes of Health, Bill and Melinda Gates Foundation and Lily and Ernst Hausmann Trust.

Conflicts of interest. None.

1. World Health Organization. Global Hearts Initiative: Working Together to Beat Cardiovascular Disease. Geneva: WHO, 2018

2. Ntusi NAB, Mayosi BM. Epidemiology of heart failure in sub-Saharan Africa. Expert Rev Cardiovasc Ther 2009;7(2):169-180. https://doi.org/10.1586/14779072.7.2.169

Sliwa K, Ntusi NAB. Battling cardiovascular disease in a perfect storm: South Africa 25 years after apartheid. Circulation 2019;139(14):1658-1660. https://doi.org/10.1161/CIRCULATIONAHA.118.038001

4. George AL. Use of contemporary genetics in cardiovascular diagnosis. Circulation 2014;130(22):19711980. https://doi.org/10.1161/CIRCULATIONAHA.114.006511

5. Fieggen K, Ntusi NAB. Understanding the genetic basis of human health and disease: Role of molecular genetics in diagnosis and prognostication. S Afr Med J 2019;109(4):204-206. https://doi.org/10.7196 SAMJ.2019.v109i4.14037

6. Marian AJ, van Rooij E, Roberts R. Genetics and genomics of single-gene cardiovascular disease: Common hereditary cardiomyopathies as prototypes of single-gene disorders. J Am Coll Cardiol 2016;68(25):2831-2849. https://doi.org/10.1016/j.jacc.2016.09.968

7. Cirino AL, Harris S, Lakdawala NK, et al. Role of genetic testing in inherited cardiovascular disease Cirino AL, Harris S, Lakdawala NK, et al. Role of genetic testing in inherited car
JAMA Cardiol 2017;2(10):1153-1160. https://doi.org/10.1001/jamacardio.2017.2352

Ackerman MI, Priori SG, Willems S, et al. HRS/EHRA expert consensus statement on the state of genetic Ackerman MJ, Priori SG, Willems S, et al. HRS/EHRA expert consensus statement on the state of genetic
testing for the channelopathies and cardiomyopathies. Europace 2011;13(8):1077-1109. https://doi. testing for the channelopathi
org/10.1093/europace/eur245

org/10.1093/europace/eur245
9. Burke MA, Cook SA, Seidman JG, Seidman CE. Clinical and mechanistic insights into the genetics of Burke MA, Cook SA, Seidman JG, Seidman CE. Clinical and mechanistic insights into the genetics of
cardiomyopathy. J Am Coll Cardiol 2016;68(25):2871-2886. https:///doi.org/10.1016/j.jacc.2016.08.079

cardiomyopathy. J Am Coll Cardiol 2016;68(25):2871-2886. https://doi.org/10.1016/j.jacc.2016.08.07
Charron P, Arad M, Arbustini E, et al. Genetic counselling and testing in cardiomyopathies: A position Charron P, Arad M, Arbustini E, et al. Genetic counselling and testing in cardiomyopathies: A position
statement of the European Society of Cardiology Working Group on Myocardial and Pericardial statement of the European Society of Cardiology Working Group on Myocardial
Diseases. Eur Heart J 2010;31(22):2715-2728. https://doi.org/10.1093/eurheartj/ehq271

Diseases. Eur Heart J 2010;31(22):2715-2728. https://doi.org/10.1093/eurheartj//hqh271
11. Johansen CT, Wang J, Lanktree MB, et al. Nat Genet 2010;42(8):684-687. https://doi.org/10.1038/ng.628

11. Johansen CT, Wang J, Lanktree MB, et al. Nat Genet 2010;42(8):684-687. https://doi.org/10.1038/ng.628
12. Kathiseran S, Srivastava D. Genetics of human cardiovascular disease. Cell 2012;148(6):1242-1257. https://doi.org/10.1016/j.cell.2012.03.001

13. Lehrman MA, Schneider WJ, Sudhof TC, Brown MS, Goldstein JL, Russell DW. Mutation in LDL receptor: Alu-Alu recombination deletes exons encoding transmembrane and cytoplasmic domains. Science 1985;227(4683):140-146.

4. Geisterfer-Lowrance AA, Kass S, Tanigawa G, et al. A molecular basis for familial hypertrophic cardiomyopathy: A beta cardiac myosin heavy chain gene missense mutation. Cell 1990;62(5):999-1006. 5. Dietz HC. New therapeutic approaches to Mendelian disorders. N Engl J Med 2010;363(9):852-863. https://doi.org/10.1056/NEJMra0907180

6. Kannel WB, Dawber TR, Kagan A, Revotskie N, Stokes J 3rd. Factors of risk in the development of coronary heart disease - six year follow-up experience. The Framingham Study. Ann Intern Med 1961;55:33-50.

7. Davies NM, Holmes MV, Smith GD. Reading Mendelian randomisation studies: A guide, a glossary and checklist for clinicians. BMJ 2018;362:k601. https://doi.org/10.1136/bmj.k601

18. Yohe S, Thyagajaran B. Review of clinical next-generation sequencing. Arch Pathol Lab Med 2017;141(11):1544-1557. https://doi.org/10.5858/arpa.2016-0501-RA

19. Bournele D, Beis D. Zebrafish models of cardiovascular disease. Heart Fail Rev 2016;21(6):803-813 https://doi.org/10.1007/s10741-016-9579-y

20. Zaragoza C, Gomez-Guerrero C, Martin-Ventura JL, et al. Animal models of cardiovascular diseases. J Biomed Biotechnol 2011;2011:497841. https://doi.org/10.1155/2011/497841

21. Ntusi N, Shaboodien G, Badri M, Gumedze F, Mayosi B. Clinical features, spectrum of causal genetic mutations and outcome of hypertrophic cardiomyopathy in South Africans. Cardiovasc J Afr 2016;27(3):152-158. https://doi.org/10.5830/CVJA-2015-075 